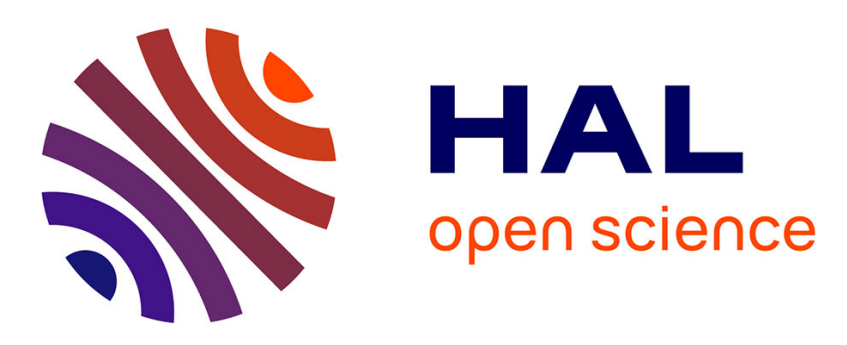

\title{
Zonotopic constrained Kalman filter based on a dual formulation
}

Dory Merhy, Teodoro Alamo, Cristina Stoica Maniu, Eduardo Camacho

\section{To cite this version:}

Dory Merhy, Teodoro Alamo, Cristina Stoica Maniu, Eduardo Camacho. Zonotopic constrained Kalman filter based on a dual formulation. 57th IEEE Conference on Decision and Control (CDC 2018), Dec 2018, Miami Beach, United States. 10.1109/cdc.2018.8619177 . hal-01907119

\section{HAL Id: hal-01907119 https://hal.science/hal-01907119}

Submitted on 16 Mar 2020

HAL is a multi-disciplinary open access archive for the deposit and dissemination of scientific research documents, whether they are published or not. The documents may come from teaching and research institutions in France or abroad, or from public or private research centers.
L'archive ouverte pluridisciplinaire HAL, est destinée au dépôt et à la diffusion de documents scientifiques de niveau recherche, publiés ou non, émanant des établissements d'enseignement et de recherche français ou étrangers, des laboratoires publics ou privés. 


\title{
Zonotopic Constrained Kalman Filter based on a Dual Formulation
}

\author{
Dory Merhy, Teodoro Alamo, Cristina Stoica Maniu, Eduardo F. Camacho
}

\begin{abstract}
This paper presents a new zonotopic constrained approach for the Kalman filter that takes advantage of the particular structure of the original optimization problem. This technique consists in projecting the state estimation by solving an optimization problem, to ensure that the estimated state belongs to a zonotope. Based on a classical gradient algorithm method, i.e. the iterative shrinkage-thresholding algorithm (ISTA), this paper proposes a reduced complexity approach suitable for the state estimation of systems subject to a large number of state constraints. The algorithm's speed is improved via a faster ISTA approach, called FISTA.
\end{abstract}

Index Terms-constrained Kalman filter, primal-dual form.

\section{INTRODUCTION}

In the state estimation theory, the Kalman filter [1] is well known to be suitable for the state estimation of linear systems assuming the knowledge of the characteristics (covariance, average, etc.) of perturbations and noises governing the state and measurements. Moreover, many extensions of the Kalman filter have been developed through time to deal with real time systems (e.g. Extended Kalman filter [2], unscented Kalman filter [3], and Kalman-Bucy filter [4]). Due to its accuracy and easy implementation, the Kalman filter is widely used in industrial fields like radar imaging [5], finance [6], and many more. As an alternative, the deterministic approach, particularly the set-membership state estimation became subject of research interest since it considers bounded perturbations and measurement noises. Additionally, the estimated state belongs to a geometrical set [7] such as ellipsoid, zonotope, polytope etc. Among these sets, zonotopes are used for state estimation purposes due to their reduced complexity, their accuracy and their facility in mathematical operations computation [8], [9]. With the intention of mixing the advantages of both approaches, in [10], the classic Kalman filter and the zonotopic state estimation are combined in a zonotopic Kalman filter (ZKF) based on the introduction of a new notion of covariation connecting the two approaches. Even though the Kalman filter is a powerful tool in state estimation, some of the known

D. Merhy and C. Stoica Maniu are with Laboratoire des Signaux et Systèmes (L2S), CentraleSupélec-CNRS-Université Paris-Sud, Université Paris-Saclay, 3 rue Joliot Curie, F91192, Gif-sur-Yvette cedex, France (e-mail: \{dory.merhy; cristina.stoica\}@l2s. centralesupelec.fr).

T. Alamo and E.F. Camacho are with Department of Ingeniería de Sistemas y Automática, Universidad de Sevilla, Camino de los Descubrimientos, 41092 Sevilla, Spain (e-mail: alamo@cartuja.us.es, eduardo@esi.us.es).

The first author acknowledges the support of the STIC Doctoral School for a 3-month visit at University of Seville. The authors acknowledge the support of MEyC Spain (contract DPI2016-76493-C3-1-R), the University of Sevilla (VI PPITUS), and the European Research Council (Advanced Research Grant 769051 OCONTSOLAR). information about the system state can not be integrated. For example, distances and speeds are always positive, and these information should be treated as state constraints on during the state estimation.

There has been a wide use of systems with state constraints in state estimation applications that include but are not limited to biomedical systems [11], camera tracking [12] and others. When the system is subject to equality constraints, different approaches can be applied to incorporate them. One approach focuses, for instance, on the system model reduction [13], decreasing the number of computations of the Kalman filter even though it might lead to the loss of the physical meaning of the state variables. Another technique is to consider the constraints as perfect measurements with zero measurement noise [14], but this will give a new augmented measurement equation leading to a singular measurement noise covariance. In addition, increasing the dimension of the problem leads to larger computational complexity of the Kalman gain. A third approach is to project the unconstrained estimation onto the constraint surface at each time iteration [15]. These three different approaches result in the same final optimal state estimation as proven in [16], in which a comparison of Kalman filters results in case of equality and inequality constraints, for the different methods, has been made. The advantage of the third approach lies in its capacity to be extended to inequality constraints. Another method to use for inequality constraints is the probability density function (PDF) truncation in which the constrained state estimate equals the mean of the truncated PDF [17], [18]. In parallel, the same problem has been treated differently in [19]: a set-based state estimation problem has been considered using constrained zonotopes to take into account linear equality constraints on the unit hypercube within its generators representation. Nevertheless, if the problem of state estimation is addressed using zonotopes, the complexity grows considerably due to set operations (e.g. intersection).

In this work, a new approach for a constrained Kalman filter is proposed guaranteeing that the estimated state, at each time instant, belongs to a given zonotope, which is the envelope of the constraints applied on the system state. Indeed, the unconstrained Kalman estimation is projected onto the zonotope, i.e. an optimization problem is solved at each time iteration such that the state belongs to this zonotope. Unlike the common deterministic approaches dealing with zonotopes, this technique requires no intersection between two geometrical sets. The main contribution of the paper is the development of a new approach for a zonotopic constrained Kalman filter (ZCKF) for systems subject to a potentially large number of linear inequality constraints on 
the state, with reduced complexity due to the use of an efficient optimization strategy. Indeed, the novelty of this paper is the design of a dual algorithm taking advantage of the particular structure of the problem. The complexity of the optimization problem will no longer be affected by the potentially large number of constraints defining the zonotope. Within the algorithm, a particularization of the iterative shrinkage-thresholding algorithm (ISTA) is presented to take advantage of its simplicity. Then, an improvement of the convergence rate is considered by using the fast ISTA algorithm, known as FISTA [20], [21].

Notation. The following notation is further used $\|x\|_{Q}^{2}=$ $x^{\top} Q x$, with a vector $x$ and a symmetric positive definite matrix $Q=Q^{\top} \succ 0$. A random variable $x$ normally distributed with mean of $\bar{x}$ and covariance matrix $G$ is represented by $x \sim N(\bar{x}, G)$. Here, $E[\cdot]$ is the expectation operator. The notation $\langle a, b\rangle$ is the scalar product between the two vectors $a$ and $b$. The Minkowski sum of the two sets $\mathcal{A}$ and $\mathcal{B}$ is denoted by $\mathcal{A} \oplus \mathcal{B}=\{a+b: a \in \mathcal{A}, b \in \mathcal{B}\}$. The set $\mathcal{Z}=(p ; H)=p \oplus H \mathbf{B}^{m}$ represents a zonotope of center $p \in \mathbb{R}^{n}$ and matrix of generators $H \in \mathbb{R}^{n \times m}$, with $m$ the order of the zonotope and $\mathbf{B}^{m}$ a unitary box in $\mathbb{R}^{n}$.

\section{Constrained Kalman Filter}

Let us consider the following discrete-time Linear Time Invariant (LTI) system

$$
\left\{\begin{aligned}
x_{k+1} & =A x_{k}+B u_{k}+w_{k} \\
y_{k} & =C x_{k}+v_{k}
\end{aligned}\right.
$$

where $x_{k} \in \mathbb{R}^{n_{x}}, u_{k} \in \mathbb{R}^{n_{u}}$ and $y_{k} \in \mathbb{R}^{n_{y}}$ are respectively the state of the system, the input vector, and the measured output vector at sample time $k$. The matrices $A, B$ and $C$, with appropriate dimensions, are respectively the system, control and output matrices. Here, $w_{k} \in \mathbb{R}^{n_{x}}$ and $v_{k} \in \mathbb{R}^{n_{y}}$ are random, independent white Gaussian noises with zero mean and covariances $G_{w}$ and $G_{v}$ respectively. Notice that the state is a random Gaussian vector and the initial state is denoted by $x_{0} \sim N\left(x_{0 \mid-1}, G_{0 \mid-1}\right)$. In this context, given an estimation for $x_{k}$ subject to known linear constraints, the objective of this paper is to provide a zonotopic constrained estimation for $x_{k+1}$. In other terms, the envelope of the constraints is a zonotope of the form $\mathcal{Z}=(p ; H)$.

The Kalman filter is a recursive estimator, conceptualized in two phases: prediction and correction. The first phase uses the estimation from a previous time instant $\hat{x}_{k-1 \mid k-1}$ to produce a new estimate of the state at the current instant $\hat{x}_{k \mid k-1}$. In the second phase, the prediction is combined with the measurement information $y_{k}$ to refine the estimation $\hat{x}_{k \mid k}$. The Kalman filter equations are:

$$
\begin{aligned}
\hat{x}_{k \mid k-1} & =A \hat{x}_{k-1 \mid k-1}+B u_{k-1} \\
G_{k \mid k-1} & =A G_{k-1 \mid k-1} A^{\top}+G_{w} \\
S_{k} & =C G_{k \mid k-1} C^{\top}+G_{v} \\
K_{k} & =G_{k \mid k-1} C^{\top} S_{k}^{-1} \\
\hat{x}_{k \mid k} & =\hat{x}_{k \mid k-1}+K_{k}\left(y_{k}-C \hat{x}_{k \mid k-1}\right) \\
G_{k \mid k} & =\left(I-K_{k} C\right) G_{k \mid k-1}
\end{aligned}
$$

with $K_{k}$ the Kalman gain and $S_{k}$ the innovation covariance at the sample time $k$. It should be noticed that $G_{k \mid k}$ is the covariance of the estimation error $x_{k}-\hat{x}_{k \mid k}$.

Unavailable in the classical filter, constrained filtering consists on correcting the estimation by considering constraints on the state vector [16]. Suppose that the system verifies the inequality constraints $\tilde{K} x_{k} \leq \tilde{c}$ with the matrix $\tilde{K}$ and the vector $\tilde{c}$ of appropriate dimensions. The constrained estimate is obtained solving the following optimization problem

$$
\begin{array}{ll}
\min _{x_{k}} & \left\|x_{k}-\hat{x}_{k \mid k}\right\|_{W}^{2} \\
\text { s.t. } & \tilde{K} x_{k} \leq \tilde{c}
\end{array}
$$

where $W$ is a symmetric positive-definite weighting matrix $W=W^{\top} \succ 0$. If $W=G_{k \mid k}^{-1}$, the maximum probability estimate of the state w.r.t. constraints is obtained [15]. The constrained estimate is the solution of a quadratic programming problem. For general quadratic optimization problems, various methods are commonly used. For example, the interior point method [22] solves inequality constrained convex problems in polynomial time, whereas the active set method [23] proceeds by solving equality subproblems and verifying if the original constraints are satisfied.

The algorithm in this paper is based on a dual formulation of the (primal) problem of obtaining the minimum weighted distance of the unconstrained Kalman estimation to a zonotopic constraint. The designed algorithm belongs to the class of accelerated gradient methods [24], [21]. It allows us to address zonotopic constraints, in the context of the Kalman filter, with a simple implementation that is well suited for real time applications. The presented algorithm inherits the convergence properties of accelerated methods.

\section{ZONOTOPIC CONSTRAIned KaLMAN FILTER}

Motivated by the above discussion, the main result of our paper is detailed in this section.

\section{A. Prerequisites on zonotopes}

A polytope can be defined as a bounded intersection of a finite number of half-spaces

$$
\mathcal{P}=\left\{x \in \mathbb{R}^{n_{x}}: K x \leq c\right\},
$$

where $K \in \mathbb{R}^{m \times n_{x}}$ and $c \in \mathbb{R}^{m}$. A zonotope is the linear projection of a unitary $m$-dimensional hypercube

$$
\mathcal{Z}=(p ; H)=p \oplus H \mathbf{B}^{m} \text {. }
$$

The vector $p \in \mathbb{R}^{n_{x}}$ denotes its center and $H=$ $\left[\begin{array}{llll}h_{1} & h_{2} & \ldots & h_{m}\end{array}\right] \in \mathbb{R}^{n_{x} \times m}$ is called the matrix of generators. A zonotope is a symmetric convex polytope that can also be represented by the half-space representation. The conversion between the two representations is studied in [25], [26]. Notice that solving a convex optimization problem, constrained by a zonotope, is equivalent to solving an optimization problem with linear inequalities. Obtaining the optimal solution using classical optimization strategies, specially when the zonotope has a large number of generators, can be quite time consuming and not well suited 
for online implementations. The following subsections show how to implement a dual formulation of the original problem that takes into consideration the specific structure of the zonotopic constraint. In the dual formulation the number of decision variables grows with the dimension of the statespace and not with the number of generators required to define the zonotopic constraint.

\section{B. Preliminary work}

In the context of the duality principle, optimization problems can be seen from two perspectives: the primal and the dual problem. In a convex problem, the primal and dual optimal objective values are equal under rather general assumptions [27]. In this work, we address an optimization problem of the form

$$
\begin{aligned}
& J^{*}=\min _{u \in U} J_{u}(u) \\
& \text { s.t. } \quad R u-p=0 \text {. }
\end{aligned}
$$

It is assumed that $U \subseteq \mathbb{R}$ is a smooth strictly convex function. Following the dual approach proposed in Nesterov's work [28], a formal definition of the dual cost is provided.

Definition 1: Given the matrix $R \in \mathbb{R}^{n \times m}$, the vector $p \in$ $\mathbb{R}^{n}$, and the primal function $J_{u}: U \rightarrow \mathbb{R}$ from (11), let us define the smooth function called dual function

$$
f(\alpha)=\max _{u \in U}\langle\alpha, R u-p\rangle-J_{u}(u)
$$

and

$$
u(\alpha)=\arg \max _{u \in U}\langle\alpha, R u-p\rangle-J_{u}(u) .
$$

Following the results in [28], given that

$$
-J^{*} \leq \max _{u \in U}\langle\alpha, R u-p\rangle-J_{u}(u)
$$

with $J^{*}$ defined by (11), the solution of the original problem is obtained by minimizing $f(\alpha)$ with respect to $\alpha$, with an inner maximization problem to find the optimal value of $u(\alpha)$. For this formulation, the following property holds.

Property 1: [28] Suppose that $J_{u}(\cdot)$ is a strictly convex function with the hessian $\frac{\partial^{2} J_{u}}{\partial u^{2}}(u) \succeq S \succ 0$ and that $U$ is a convex set. Then, with $\Delta \alpha=\alpha-\bar{\alpha}$, denoting

$$
\bar{u}=u(\bar{\alpha})=\arg \max _{u \in U}\langle\bar{\alpha}, R u-p\rangle-J_{u}(u),
$$

we have

$$
f(\alpha) \leq f(\bar{\alpha})+\langle R \bar{u}-p, \Delta \alpha\rangle+\frac{1}{2} \Delta \alpha^{\top} R S^{-1} R^{\top} \Delta \alpha
$$

This property states that in order to compute an increment $\Delta \alpha$ leading to an improvement of the dual function (i.e. $f(\alpha+\Delta \alpha) \leq f(\alpha))$ it suffices to obtain $\Delta \alpha$ in such a way that

$$
\langle R \bar{u}-p, \Delta \alpha\rangle+\frac{1}{2} \Delta \alpha^{\top} R S^{-1} R^{\top} \Delta \alpha
$$

is minimized. The optimal value for $\Delta \alpha$ has an explicit solution because the function to be minimized is a quadratic convex function of $\Delta \alpha$ (as detailed in Subsection III-D).

\section{Algorithmic solution for ZCKF}

The state estimation of the system (1) at each time instant $k$ is subject to a system of inequality constraints that can be rewritten as a zonotopic constraint of the form $x_{k} \in p \oplus$ $H \mathbf{B}^{m}$. This constraint is equivalent to the existence of $w \in$ $\mathbb{R}^{m}$ such that $\|w\|_{\infty} \leq 1$ and $x_{k}=p+H w$, with $p \in$ $\mathbb{R}^{n_{x}}$ the center and $H=\left[\begin{array}{llll}h_{1} & h_{2} & \ldots & h_{m}\end{array}\right] \in \mathbb{R}^{n_{x} \times m}$ the generators matrix of the zonotope. Hence, we are faced to the problem of computing the minimum distance from the unconstrained Kalman estimate $\hat{x}_{k \mid k}$ to the zonotope. This distance is zero if $\hat{x}_{k \mid k}$ is included in the zonotope. The constrained Kalman filter optimization problem is

$$
\begin{array}{ll}
\min _{z, w} & J_{z, w}(z, w) \\
\text { s.t. } & z=p+H w \\
& \|w\|_{\infty} \leq 1
\end{array}
$$

where the cost function is defined such as

$$
\begin{aligned}
J_{z, w}(z, w) & =J_{z}(z)+\frac{\epsilon}{2} w^{\top} w= \\
& =\frac{1}{2}\left\|z-\hat{x}_{k \mid k}\right\|_{G_{k \mid k}^{-1}}^{2}+\frac{\epsilon}{2} w^{\top} w .
\end{aligned}
$$

In this setting, the scalar $\epsilon>0$ is an arbitrarily small regularization parameter that guarantees that the quadratic function $J_{z, w}(z, w)$ meets the strict convexity assumption of the Property 1. From the inspection of the quadratic function $J_{z, w}(z, w)$ we have that the hessian is given by $S=\operatorname{diag}\left(G_{k \mid k}^{-1}, \epsilon I_{m}\right)$. Notice that $z=p+H w$ is equivalent to $\left[\begin{array}{ll}I & -H\end{array}\right]\left[\begin{array}{ll}z^{\top} & w^{\top}\end{array}\right]^{\top}=p$. This allows us to rewrite the problem (18) in the form of (11), with $u=\left[\begin{array}{ll}z^{\top} & w^{\top}\end{array}\right]^{\top}$, $R=\left[\begin{array}{ll}I & -H\end{array}\right]$ and $J_{u}(u)=J_{u}\left(\left[\begin{array}{c}z \\ w\end{array}\right]\right)=J_{z, w}(z, w)$. The function $u(\alpha)$, as defined in Property 1 , is given by $u(\alpha)=\arg \max _{\|w\|_{\infty} \leq 1, z \in \mathbb{R}^{n_{x}}}\langle\alpha, z-p-H w\rangle-J_{z}(z)-\frac{\epsilon}{2} w^{\top} w$. We notice that the previous optimization problem can be decomposed in two independent ones as shown in (20)

$$
u(\alpha)=\left[\begin{array}{c}
z(\alpha) \\
w(\alpha)
\end{array}\right]=\left[\begin{array}{c}
\arg \max _{z \in \mathbb{R}^{n_{z}}}\langle\alpha, z\rangle-J_{z}(z) \\
\arg \max _{\|w\|_{\infty} \leq 1}-\langle\alpha, H w\rangle-\frac{\epsilon}{2} w^{\top} w
\end{array}\right]_{(20)}
$$

As noticed, the variable $z$, constrained by the linear inequalities forming the zonotope in the primal mode, belongs to the set of real numbers in the dual mode. In other terms, the number of decision variables in the optimization problem no longer depends on the number of linear constraints, but on the dimension of the state-space. As it is detailed in subsection III-D, given $\alpha_{j}$, both $z\left(\alpha_{j}\right)=z_{j}$ and $w\left(\alpha_{j}\right)=$ $w_{j}=\left[\begin{array}{llll}w_{j}(1) & w_{j}(2) & \ldots & w_{j}(m)\end{array}\right]^{\top}$ can be obtained from the following closed explicit expressions

$$
\begin{gathered}
z_{j}=G_{k \mid k} \alpha_{j}+\hat{x}_{k \mid k} . \\
w_{j}(i)=\left\{\begin{array}{ll}
-\frac{1}{\epsilon} \alpha_{j}^{\top} h_{i}, & \text { if }\left|\frac{1}{\epsilon} \alpha_{j}^{\top} h_{i}\right| \leq 1 \\
1, & \text { if }-\frac{1}{\epsilon} \alpha_{j}^{\top} h_{i}>1 \\
-1, & \text { if }-\frac{1}{\epsilon} \alpha_{j}^{\top} h_{i}<-1
\end{array}, i=1, \ldots, m .\right.
\end{gathered}
$$


In view of Property 1 we have that the gradient of the dual cost for a given $\alpha_{j}$ is given by $R u_{j}-p=z_{j}-p-$ $H w_{j}$. Moreover, Property 1 also states that an optimal local improvement with respect to the value obtained for $\alpha_{j}$ is given by $\alpha_{j}+\Delta \alpha_{j}$, where $\Delta \alpha_{j}$ minimizes

$$
\left\langle R u\left(\alpha_{j}\right)-p, \Delta \alpha_{j}\right\rangle+\frac{1}{2} \Delta \alpha_{j}^{\top} R S^{-1} R^{\top} \Delta \alpha_{j} .
$$

As it is also detailed in subsection III-D, given $z_{j}$ and $w_{j}$, the optimal increment $\Delta \alpha_{j}$ is given by

$$
\Delta \alpha_{j}=\left(R S^{-1} R^{\top}\right)^{-1}\left(p+H w_{j}-z_{j}\right) .
$$

Notice that this last expression provides an exit condition for an algorithm based on the gradient information. If the norm of $p+H w_{j-1}-z_{j-1}$ is small, the pair $w_{j-1}$ and $z_{j-1}$ is close to optimality because the gradient at $\alpha_{j-1}$ is close to zero. Therefore, the norm of $\left\|p+H w_{j-1}-z_{j-1}\right\|$ could be used as exiting condition for a numerical algorithm computing the solution of the zonotopic constrained Kalman estimation.

We present in what follows (see Algorithm 1) the particularization of the iterative shrinkage-thresholding algorithm (ISTA) to the specific dual formulation adopted in this paper.

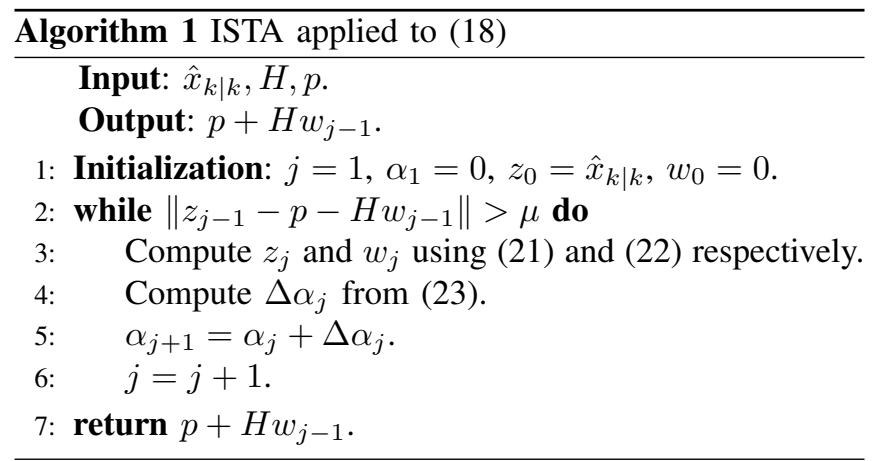

The variable $\mu$ denotes the desired tolerance for which the solution is finally reached. Notice that the output $p+H w_{j-1}$ belongs, by construction, to the zonotope $p \oplus H \mathbf{B}^{m}$. Moreover, $p+H w_{j-1}$ provides, up to a numeric accuracy controlled by the exit parameter $\mu$, the closest point, according to the weighted norm $\|\cdot\|_{G_{k \mid k}^{-1}}$, to the original unconstrained Kalman estimation $\hat{x}_{k \mid k}$. We conclude that the output of the algorithm provides the numerical solution to the zonotopic Kalman filter estimation problem.

The convex optimization problem (18) could be solved via classical methods. However, with high order zonotopes, the problem can involve a large number of constraints, which motivates the use of our approach. The advantage of the algorithm above is its simplicity. However, the ISTA algorithm is recognized as a slow method [24], [21]. In fact, it is guaranteed to converge with a convergence rate of $\mathcal{O}\left(\frac{1}{j}\right)$. A faster algorithm, called fast iterative shrinkage-thresholding algorithm (FISTA) provides a better convergence rate: the difference with the optimal solution decreases with $\mathcal{O}\left(\frac{1}{j^{2}}\right)$, see [24], [21]. The speed of convergence and simplicity of both algorithms make them well suited for online applications [29]. In what follows, we present how to adapt the
FISTA algorithm to the optimization problem considered in this paper (see Algorithm 2). The main difference between the two algorithms relies in the fact that in FISTA, the gradient of the dual function is evaluated as a linear combination of the last two values of an auxiliary variable $\eta_{j}$ [21]. This means that $\alpha_{j+1}$ is obtained by interpolation between the two previous points of the auxiliary variable $\left(\eta_{j}\right.$ and $\left.\eta_{j-1}\right)$. The computational time per iteration for FISTA is basically the same as for ISTA. However, as commented before, the convergence rate of FISTA is much better. The convergence analysis of both algorithms is extensively detailed in [21].

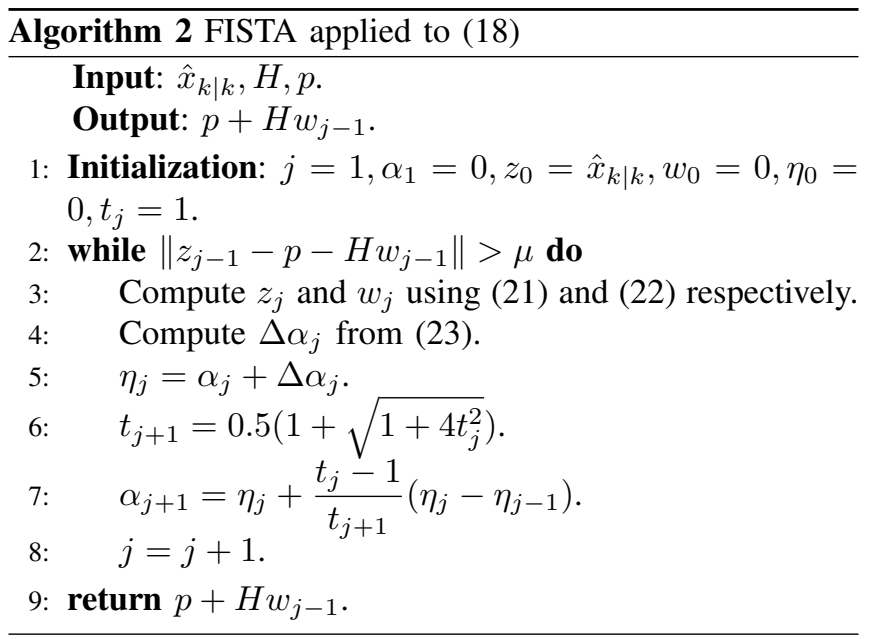

\section{Computation details}

This subsection details how to obtain the explicit expressions for $z_{j}, w_{j}$ and $\Delta \alpha_{j}$, required to implement both ISTA and FISTA algorithms.

1) Expression for $z\left(\alpha_{j}\right)$ : Based on expression (20), we recall here the definition of $z_{j}=z\left(\alpha_{j}\right)$

$$
z_{j}=\arg \max _{z \in \mathbb{R}^{n}}\langle\alpha, z\rangle-J_{z}(z)
$$

where $J_{z}(z)=\frac{1}{2}\left\|z-\hat{x}_{k \mid k}\right\|_{G_{k \mid k}^{-1}}^{2}$. Denoting $\Delta z_{j}=z_{j}-\hat{x}_{k \mid k}$, equation (24) becomes $z_{j}=\hat{x}_{k \mid k}+\Delta z_{j}$, where

$$
\Delta z_{j}=\arg \max _{\Delta z \in \mathbb{R}^{n} z} \alpha_{j}^{\top} \Delta z-\frac{1}{2} \Delta z^{\top} G_{k \mid k}^{-1} \Delta z .
$$

In order to obtain $\Delta z_{j}$ we have to determine the value for $\Delta z$ that cancels the gradient, i.e. $\Delta z_{j}$ is given by

$$
\alpha_{k}-G_{k \mid k}^{-1} \Delta z_{j}=0 .
$$

Thus, $\Delta z_{j}=G_{k \mid k} \alpha_{j}$ and then $z_{j}$ is obtained by (21).

2) Expression for $w\left(\alpha_{j}\right)$ : We recall from (20) that

$$
w\left(\alpha_{j}\right)=\arg \max _{\|w\|_{\infty} \leq 1}-\left\langle\alpha_{j}, p+H w\right\rangle-\frac{\epsilon}{2} w^{\top} w .
$$

Denote $w_{j}=w\left(\alpha_{j}\right)=\left[\begin{array}{llll}w_{j}(1) & w_{j}(2) & \ldots & w_{j}(m)\end{array}\right]^{\top}$. We show in what follows that it is possible to obtain each component of $w_{j}$ from the solution of a one dimensional optimization problem. Since $H=\left[\begin{array}{llll}h_{1} & h_{2} & \ldots & h_{m}\end{array}\right]$, we have from (26) 


$$
\begin{gathered}
w_{j}=\arg \max _{w(i), i=1, \ldots, m}-\sum_{i=1}^{m}\left(\alpha_{j}^{\top} h_{i}\right) w(i)+\sum_{i=1}^{m} \frac{\epsilon}{2} w(i)^{2} \\
\text { s.t. }|w(i)| \leq 1, i=1, \ldots, m .
\end{gathered}
$$

We notice that this is a separable optimization problem in which each component $w_{j}(i)$ can be obtained from

$$
w_{j}(i)=\arg \max _{w \in \mathbb{R},|w| \leq 1}-\left(\alpha_{j}^{\top} h_{i}\right) w+\frac{\epsilon}{2} w^{2}, i=1, \ldots, m .
$$

We remark that the gradient of $-\left(\alpha_{j}^{\top} h_{i}\right) w+\frac{\epsilon}{2} w^{2}$ vanishes at $-\frac{1}{\epsilon} \alpha_{j}^{\top} h_{i}$. This, along with the constraint $|w| \leq 1$, gives the expression (22) for each component $w_{j}(i)$

3) Expression for $\Delta \alpha_{j}$ : The optimal local increment $\Delta \alpha_{j}$ is obtained from the minimization of $\Delta \alpha_{j}^{\top}\left(R u\left(\alpha_{j}\right)-p\right)+$ $\frac{1}{2} \Delta \alpha_{j}^{\top} R S^{-1} R^{\top} \Delta \alpha_{j}$. By deriving w.r.t. to $\Delta \alpha_{j}$ and setting the derivative equal to 0 , we obtain that the value of $\Delta \alpha_{j}$ at each iteration given by the expression (23). In this subsection it has been shown that the computation of each of the steps for both ISTA and FISTA are given by very simple expressions. The next section provides numerical examples.

\section{ILLuStRative EXAMPLES}

\section{A. First example}

Given a zonotope $\mathcal{Z}\left(p_{1}, H_{1}\right)$ with normalized random values $p_{1}=\operatorname{randn}\left(n_{x}, 1\right)$ and $H_{1}=\frac{1}{m} \operatorname{randn}\left(n_{x}, m\right)$, with $n_{x}=2$ and $m=15$, the idea is to find the closest point to the state estimate that belongs to the zonotope. The exact values of $p_{1}$ and $H_{1}$ are $p_{1}=\left[\begin{array}{ll}0.0423 & -0.0403\end{array}\right]^{\top}$ and $H_{1}=\left[\begin{array}{ccc}H_{a} & H_{b} & H_{c}\end{array}\right]$, with

$$
\begin{aligned}
H_{a} & =\left[\begin{array}{ccccc}
-0.0434 & 0.0381 & -0.1089 & 0.0431 & 0.0640 \\
0.0260 & -0.0768 & 0.0338 & 0.0086 & 0.0777
\end{array}\right] \\
H_{b} & =\left[\begin{array}{ccccc}
-0.1026 & 0.0081 & 0.0253 & 0.0524 & 0.0248 \\
-0.0480 & 0.0519 & 0.0451 & -0.0098 & -0.0081
\end{array}\right] \\
H_{c} & =\left[\begin{array}{ccccc}
-0.0299 & -0.1230 & -0.0699 & 0.0499 & -0.0972 \\
-0.0708 & 0.0315 & 0.0630 & 0.0703 & -0.0277
\end{array}\right]
\end{aligned}
$$

To test the algorithm and the assumption that the FISTA algorithm has a better convergence rate than the ISTA algorithm [21], we assume the prior knowledge of the optimal solution $z^{*}$ of the problem (18). Therefore, let us consider the Kalman state estimation to be $\hat{x}_{k \mid k}=\left[\begin{array}{ll}-1.5639 & 0.2457\end{array}\right]^{\top}$, the covariance of estimation $G_{k \mid k}=I_{n_{x}}$ at the time instant $k$, with $\epsilon=10^{-4}$ in (19) and the optimal solution that should be reached $z^{*}=\left[\begin{array}{ll}-0.8148 & -0.0702\end{array}\right]^{\top}$. Figure 1 shows that starting with the unconstrained estimate represened by the black asterisk (sub-figure 1a), the algorithm offers a new feasible point (denoted by a red circle) at each iteration, with a decreased value of the original objective function (18), which means a closer point to the blue zonotope (see sub-figures $1 \mathrm{~b}$ to $1 \mathrm{f}$ ). This was done by taking one step in the direction of the gradient of the objective function. By adopting the estimate projection method, we are computing the minimum distance from the unconstrained estimate to the zonotope. At the end of the algorithm, the last red circle reaches the pre-calculated optimal solution represented by the green asterisk. The algorithm is expected to find better and better solution until the convergence criterion is met (see
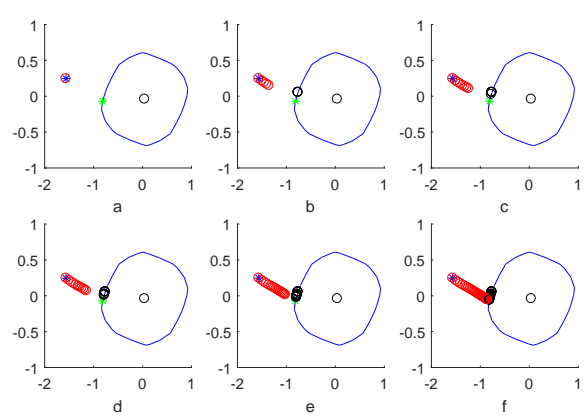

Fig. 1. Zonotopic constrained algorithm

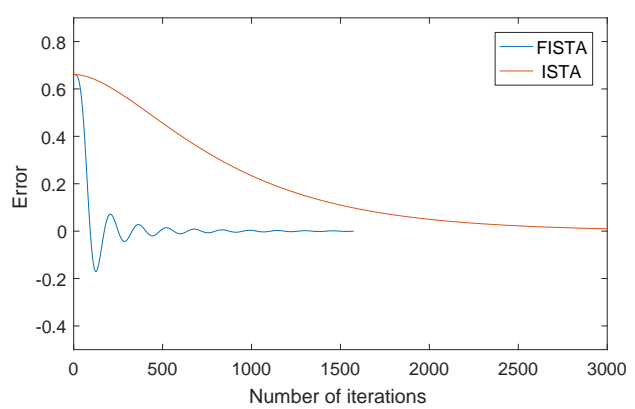

Fig. 2. Comparison of errors by the ISTA and FISTA methods

Fig. 1). The stopping criterion is the norm of the deviation of $z_{j}$ from the closest vertex of the zonotope $p+H w_{j}$ (black circle at each iteration) that is $\left\|z_{j}-p-H w_{j}\right\|$. This norm is measured at each iteration, and the algorithm terminates when it is less or equal to $\mu=10^{-8}$.

In order to expose the advantages of FISTA over ISTA, the algorithms were tested on the same example. Figure 2 shows a comparison of the function value error $J\left(z_{j}\right)-J\left(z^{*}\right)$. Clearly, the results provided by FISTA are much better than the ones provided by the ISTA algorithm in means of convergence rate. In fact, it can be seen that after 500 iterations, FISTA reaches an accuracy of $10^{-4}$, which is more precise than ISTA by several orders of magnitude, and demonstrates its efficiency. Moreover, ISTA needed more than 3000 iterations to reach the optimal value which was equivalent to 0.08 s that FISTA obtained after less than 1000 iterations (0.02s) with an accuracy of $10^{-7}$.

\section{B. Second example}

Consider the system (1) with $A=\left[\begin{array}{cc}1 & 0.3 \\ -0.225 & 0.925\end{array}\right]$, $B=\left[\begin{array}{ll}1 & 1\end{array}\right]^{\top}$ and $C=\left[\begin{array}{ll}1 & 0\end{array}\right]$. The system state is estimated using a classical Kalman filter with $x_{0} \sim$ $N\left(x_{0 \mid-1}, G_{0 \mid-1}\right), \quad u_{k}=5, w_{k} \sim N(0,0.02), v_{k} \sim$ $N(0,0.01), x_{0 \mid-1}=\left[\begin{array}{ll}0 & 2\end{array}\right]^{\top}$ and $G_{0 \mid-1}=I_{2}$. The constraints envelope (i.e. $\mathcal{Z}\left(p_{2}, H_{2}\right)$, with $p_{2}=\left[\begin{array}{ll}2 & -0.5\end{array}\right]^{\top}$ and $\left.H_{2}=\left[\begin{array}{ccc}-2 & 1 & -0.6 \\ 0.8 & -0.8 & 1.6\end{array}\right]\right)$ is chosen randomly, on the basis of knowledge of the trajectory of the system for illustration 


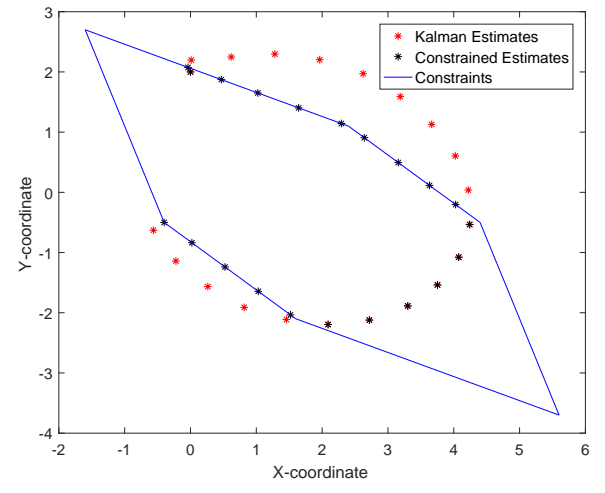

Fig. 3. Classic Kalman filter and zonotopic constrained Kalman filter

purposes. The chosen constraints are not too loose to prove the efficiency of the algorithm no matter where the estimate is located, and not too tight to keep a realistic problem, allowing variations on the state variables and trajectory. The regularization parameter is $\epsilon=10^{-4}$. Figure 3 shows the plot of the system trajectory, along with the zonotope representing the constraints envelope, the unconstrained estimates and the constrained estimates. The red asterisks obtained by applying the classical Kalman filter, are the input for the constraints algorithm. It can be noticed that the unconstrained estimates remain the same if they already belong to the zonotope (blue lines). If not, the constrained estimates (black asterisks) are obtained using the proposed algorithm. The constrained estimates, then, belong to the zonotope.

\section{CONCLUSION}

In this paper we have presented a new approach for designing a zonotopic constrained Kalman filter. The proposed technique is able to guarantee, at every time instant, that the current system state belongs to a zonotope. The state estimation design is based on projecting the unconstrained estimated state on a zonotope, by solving an optimization problem based on the dual formulation of the original optimization problem. Due to its simplicity, the iterative shrinkage-thresholding algorithm (ISTA) is used to find an optimal solution. An improvement of the convergence rate of the algorithm has been further presented by using the fast ISTA algorithm. Two numerical examples have been provided to illustrate the effectiveness of the proposed techniques. Current work consists in applying the proposed zonotopic constrained Kalman approach to a real system.

\section{REFERENCES}

[1] R. E. Kalman, "A new approach to linear filtering and prediction problems," Transactions of the ASME-Journal of Basic Engineering, vol. 82, no. Series D, pp. 35-45, 1960.

[2] S. F. Schmidt, "Application of state-space methods to navigation problems." Elsevier, 1966, vol. 3, pp. 293-340.

[3] E. A. Wan and R. V. D. Merwe, "The unscented Kalman filter for nonlinear estimation," in IEEE Adaptive Systems for Signal Processing, Communications, and Control Symposium, 2000, pp. 153-158.

[4] R. E. Kalman and R. S. Bucy, "New results in linear filtering and prediction theory," Transactions of the ASME, Ser. D, J. Basic Eng, p. 109,1961
[5] T. Chevet, M. Makarov, C. Stoica Maniu, I. Hinostroza, and P. Tarascon, "State estimation of an octorotor with unknown inputs. Application to radar imaging," in 21st International Conference on System Theory, Control and Computing (ICSTCC), 2017, pp. 723-728.

[6] M. Manoliu and S. Tompaidis, "Energy futures prices: term structure models with Kalman filter estimation," Applied Mathematical Finance, vol. 9, no. 1, pp. 21-43, 2002

[7] S. Ben Chabane, C. Stoica Maniu, T. Alamo, E. F. Camacho, and D. Dumur, "Improved set-membership estimation approach based on zonotopes and ellipsoids," in Proc. of European Control Conference, Strasbourg, France, 2014

[8] T. Alamo, J. M. Bravo, and E. F. Camacho, "Guaranteed state estimation by zonotopes," Automatica, vol. 41, pp. 1035-1043, 2005.

[9] C. Combastel, "A state bounding observer based on zonotopes," in Proc. of European Control Conference, Cambridge, UK, 2003.

[10] C. Combastel, "Zonotopes and Kalman observers: Gain optimality under distinct uncertainty paradigms and robust convergence," Automatica, vol. 55, pp. 265-273, 2015.

[11] V. Quintana, B. Scott, and A. Chikhani, "Constrained power system state estimation," 10th IFAC Triennial World Congress on Automatic Control, Germany.

[12] S. J. Julier and J. J. LaViola, "On Kalman filtering with nonlinear equality constraints," IEEE Transactions on Signal Processing, vol. 55, no. 6, pp. 2774-2784, 2007.

[13] W. Wen and H. F. Durrant-Whyte, "Model-based multi-sensor data fusion," in IEEE International Conference on Robotics and Automation, 1992, pp. $1720-1726$ vol.2.

[14] J. Porrill, "Optimal combination and constraints for geometrical sensor data," International Journal of Robotics Research, vol. 7, no. 6, pp. 66-77, 1988

[15] D. Simon and T. L. Chia, "Kalman filtering with state equality constraints," IEEE Transactions on Aerospace and Electronic Systems, vol. 38, no. 1, pp. 128-136, 2002.

[16] D. Simon, "Kalman filtering with state constraints: a survey of linear and nonlinear algorithms," IET Control Theory Applications, vol. 4 no. 8, pp. 1303-1318, 2010

[17] D. Simon and D. L. Simon, "Constrained Kalman filtering via density function truncation for turbofan engine health estimation," International Journal of Systems Science, vol. 41, no. 2, pp. 159-171, 2010.

[18] N. Shimada, Y. Shirai, Y. Kuno, and J. Miura, "Hand gesture estimation and model refinement using monocular camera-ambiguity limitation by inequality constraints," in Third IEEE International Conference on Automatic Face and Gesture Recognition, 1998, pp. 268-273.

[19] J. K. Scott, D. M. Raimondo, G. R. Marseglia, and R. D. Braatz, "Constrained zonotopes," Automatica, vol. 69, no. C, pp. 126-136, 2016.

[20] Y. Nesterov, "A method for unconstrained convex minimization problem with the rate of convergence $\mathrm{O}\left(1 / k^{2}\right)$," Doklady AN USSR, vol 269 , pp. 543-547, 1983 .

[21] A. Beck and M. Teboulle, "A fast iterative shrinkage-thresholding algorithm for linear inverse problems," 2009.

[22] K. A. McShane, C. L. Monma, and D. Shanno, "An implementation of a primal-dual interior point method for linear programming," ORSA Journal on Computing, vol. 1, no. 2, pp. 70-83, 1989.

[23] E. R. Panier, "An active set method for solving linearly constrained nonsmooth optimization problems," Mathematical Programming, vol. 37, no. 3, pp. 269-292, 1987.

[24] Y. Nesterov, Introductory Lectures on Convex Optimization: A Basic Course, 2003

[25] K. Fukuda, "From the zonotope construction to the Minkowski addition of convex polytopes," Journal of Symbolic Computation, vol. 38 no. 4, pp. 1261-1272, 2004.

[26] P. D. Seymour, "A note on hyperplane generation," Combinatorial Theory Series B, vol. 61, no. 1, pp. 88-91, 1994.

[27] S. Boyd and L. Vandenberghe, Convex Optimization. New York, NY, USA: Cambridge University Press, 2004.

[28] Y. Nesterov, "Smooth minimization of non-smooth functions," Mathematical Programming, vol. 103, no. 1, pp. 127-152, 2005.

[29] S. Richter, C. N. Jones, and M. Morari, "Computational complexity certification for real-time MPC with input constraints based on the fast gradient method," IEEE Transactions on Automatic Control, vol. 57 , no. 6, pp. 1391-1403, 2012. 